\section{Revista de la \\ Universidad del Thulia}

Fundada en 1947 por el Dr. Jesúns Enrique Lossada

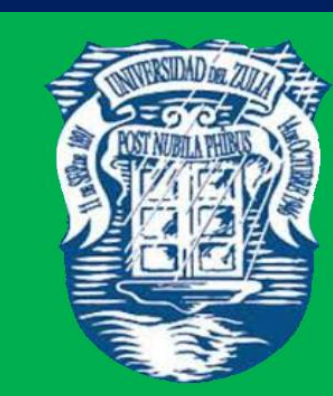

Ciencias del

Algreo

Ingemieria

y Teemología

\section{Aกัต 11 No 29}

Enero - Abril 2021

Tercera Época

Maracaibo-Venezuela 


\title{
Análisis de los sitios web de las Universidades Nacionales Interculturales Peruanas
}

\author{
Carlos Alberto Ríos Campos * \\ Nemesio Santamaría Baldera ** \\ Ronald Omar Estela Urbina ** \\ Oscar Esteban García Grados *** \\ Iván Adrianzén Olano *** \\ RESUMEN
}

El artículo se elaboró con el objetivo de realizar un análisis de los sitios web de las universidades nacionales interculturales peruanas, las cuales son cuatro y se hallan ubicadas en diversas regiones del Perú y cuentan con un sitio web activo. Los resultados indican que los sitios web de las cuatro universidades interculturales, ofrecen un canal de comunicación gratuito y rápido con la comunidad universitaria. Se concluyó que, el mayor nivel de errores (5l) y advertencias (98) fue el sitio web de la Universidad Nacional Intercultural de la Amazonía (UNIA), mientras que el menor nivel de errores (5) y advertencias (1) fue el sitio web de la Universidad Nacional Intercultural de Quillabamba (UNIQ). El mayor puntaje y rendimiento lo tiene la Universidad Nacional Intercultural de Quillabamba (UNIQ) con 78 puntos. El mayor nivel de posicionamiento lo obtuvo la Universidad Nacional Intercultural de Quillabamba (UNIQ), con 5,9 puntos. El mayor nivel de movilidad lo obtuvo la Universidad Nacional Intercultural de la Selva Central Juan Santos Atahualpa (UNISCJSA) con 4,4 puntos. El mayor nivel de experiencia de usuario lo obtuvieron la Universidad Nacional Intercultural de la Amazonía (UNIA) y la Universidad Nacional Intercultural Fabiola Salazar Leguía de Bagua (UNIBAGUA) con 6,4 puntos. El mejor rendimiento lo tiene el sitio web de la Universidad Nacional Intercultural de Quillabamba (UNIQ) con 30 puntos. El mejor tiempo de carga lo tiene el sitio web de la Universidad Nacional Intercultural de la Selva Central Juan Santos Atahualpa (UNISCJSA) con $4.9 \mathrm{~s}$.

PALABRAS CLAVE: Sitio web; TIC; universidad nacional intercultural; interculturalidad.

*Docente universitario. Universidad César Vallejo, Perú. carlosrioscampos@gmail.com

**Docente. Universidad Nacional Intercultural Fabiola Salazar Leguía de Bagua.

***Docente principal. Universidad Nacional Toribio Rodríguez de Mendoza. 


\section{Analysis of the websites of the Peruvian National Intercultural Universities}

ABSTRACT

The article was prepared with the objective of carrying out an analysis of the websites of the Peruvian intercultural national universities, which are four and are located in various regions of Peru and have an active website. The results indicate that the websites of the four intercultural universities offer a free and fast communication channel with the university community. It was concluded that the highest level of errors (51) and warnings (98) was the website of the National Intercultural University of the Amazon (UNIA), while the lowest level of errors (5) and warnings (1) was the website of the National Intercultural University of Quillabamba (UNIQ). The highest score and performance is the National Intercultural University of Quillabamba (UNIQ) with 78 points. The highest level of positioning was obtained by the National Intercultural University of Quillabamba (UNIQ), with 5.9 points. The highest level of mobility was obtained by the National Intercultural University of the Central Jungle Juan Santos Atahualpa (UNISCJSA) with 4.4 points. The highest level of user experience was obtained by the National Intercultural University of the Amazon (UNIA) and the National Intercultural University Fabiola Salazar Leguía de Bagua (UNIBAGUA) with 6.4 points. The best performance is the website of the National Intercultural University of Quillabamba (UNIQ) with 30 points. The best loading time is the website of the National Intercultural University of the Central Jungle Juan Santos Atahualpa (UNISCJSA) with $4.9 \mathrm{~s}$.

KEY WORDS: Website; TIC; national intercultural university; interculturality

\section{Introducción}

Según la UNESCO (2019), el reconocimiento de la diversidad cultural mediante una utilización innovadora de los medios y de los TIC en particular - lleva al diálogo entre civilizaciones y culturas, al respeto y a la comprensión mutua.

En las sociedades del conocimiento, las prácticas y los valores de creatividad e innovación desempeñan un rol importante, aunque sólo sea por su capacidad de poner en tela de juicio los modelos existentes para responder mejor a las nuevas necesidades de la sociedad (Aguilar, 2019; Martínez, 2019). 
REVISTA DE LA UNIVERSIDAD DEL ZULIA. 3a época. Año $11 \mathrm{~N}^{\circ}$ 29, 2020 Carlos Ríos Campos et al. /// Análisis de los sitios web de las Universidades ...307-327

El acceso a las TIC requiere de la participación de los ciudadanos, y de la sociedad en general para ayudar a generar sociedades más igualitarias, así como fortalecer los servicios de educación, salud, desarrollo económico, participación pública, disminuir la corrupción y erradicar la pobreza (Maldonado, García y Sampedro-Requena, 2019).

El uso de las TIC en la educación se ha convertido, cada vez más, en un elemento imprescindible en el entorno educativo. Este complemento, acompañado de herramientas tecnológicas ha de generar en la sociedad una realidad y presencia cada vez mayor (Hernandez, 2017).

El impacto de Internet ha sido monumental e históricamente pocos inventos en la historia de la humanidad han modificado de tal forma la vida del ser humano (Rodríguez, 2014).

Los encargados de implantar la accesibilidad en la web son los diseñadores web, que muchas veces no tienen en cuenta ni los más mínimos requisitos de accesibilidad y sus responsables y clientes tampoco son conscientes del poco esfuerzo adicional. (Fuertes \& Martínez, 2017).

En el diseño de cualquier producto, y en especial en el que aquí tratamos, los sitios web, la interfaz juega un papel primordial porque es la vía por la que el usuario se comunica con el sistema (Rovira y Marcos, 2013).

Por ende, la transparencia activa resulta ser un mecanismo que puede favorecer la comunicación e interacción entre las universidades y sus grupos de interés, más aún, cuando ésta permite llegar a diferentes partes interesadas simultáneamente (Católico-Segura, Leal-Bonilla \& Londoño-Jiménez, 2019).

La diversidad es un elemento definitorio de la dinámica de la historia y en la riqueza cultural sustentada en esas identidades se integran en su patrimonio cultural enlazadas en la trama de su tejido social (UNESCO, 2017).

La interculturalidad en la educación constituye uno de los principales objetivos propuestos en la Declaración Mundial sobre Educación Superior en el siglo XXI: Visión y Acción de la UNESCO (Alonso-García, Roque-Herrera \& Juárez-Ramos, 2019); teniendo en cuenta que, la educación intercultural es una práctica educativa 
REVISTA DE LA UNIVERSIDAD DEL ZULIA. 3a época. Año $11 \mathrm{~N}^{\circ}$ 29, 2020

Carlos Ríos Campos et al. /// Análisis de los sitios web de las Universidades ...307-327

con la que se quiere responder a la diversidad de grupos culturales para una mejor convivencia e inclusión participativa (Rueda, Paz \& Avendaño, 2019).

En el Perú, se han creado cuatro universidades nacionales interculturales. La Universidad Nacional Intercultural de la Amazonía fue creada en el año 1999 por Ley № 27250, siendo resultado de la reivindicación de los pueblos amazónicos, liderado por la Asociación Interétnica de la Selva Peruana - AIDESEP... (UNIA, 2020). Además, Espinosa (2017), afirma que:

Hacia finales del año 2010, luego de las protestas indígenas de los años 2008 y 2009 que terminaron con los trágicos incidentes del "Baguazo", el gobierno del presidente García decidió crear tres nuevas universidades interculturales: la Universidad Nacional Intercultural Juan Santos Atahualpa (UNSJA) en las provincias de Chanchamayo y Satipo (Junín), la Universidad Nacional Intercultural de Quillabamba (UNIQ) en la provincia de La Convención (Cusco), y la Universidad Nacional Intercultural Fabiola Salazar Leguía (UNIB) en la provincia de Bagua (Amazonas).

Es importante conocer como las universidades nacionales interculturales peruanas, gestionan su información a través de los sitios web. Por ello, el objetivo principal de esta investigación fue realizar un análisis de los sitios web de las universidades nacionales interculturales peruanas, teniendo como objetivos secundarios los siguientes: Determinar los problemas frecuentes que presentan los sitios web de las universidades nacionales interculturales peruanas, identificar si existen diferencias específicas entre los sitios web de las universidades nacionales interculturales peruanas y examinar las ventajas importantes que ofrecen los sitios web a las universidades nacionales interculturales peruanas.

\section{Metodología}

Las cuatro universidades nacionales interculturales peruanas, se hallan ubicadas en diversas regiones del Perú y cuentan con un sitio web activo, el cual es analizado en la presente investigación, empleando las herramientas para evaluar el sitio web. El análisis se realizó durante el mes de enero de 2020. 
REVISTA DE LA UNIVERSIDAD DEL ZULIA. 3ª época. Año 11 N² 29, 2020 Carlos Ríos Campos et al. /// Análisis de los sitios web de las Universidades ...307-327

Tabla 1. Universidades nacionales interculturales peruanas.

\begin{tabular}{|l|l|l|}
\hline \multicolumn{1}{|c|}{ Universidad } & \multicolumn{1}{c|}{ Ubicación } & \multicolumn{1}{c|}{ Url } \\
\hline $\begin{array}{l}\text { Universidad Nacional } \\
\text { Intercultural de la Selva Central } \\
\text { Juan Santos Atahualpa } \\
\text { (UNISCJSA) }\end{array}$ & $\begin{array}{l}\text { Provincias de } \\
\text { Chanchamayo y Satipo } \\
\text { (Región Junín) }\end{array}$ & http://www.uniscjsa.edu.pe \\
\hline $\begin{array}{l}\text { Universidad Nacional } \\
\text { Intercultural de Quillabamba } \\
\text { (UNIQ) }\end{array}$ & $\begin{array}{l}\text { Provincia de La } \\
\text { Convención (Región } \\
\text { Cusco) }\end{array}$ & http://www.uniq.edu.pe \\
\hline $\begin{array}{l}\text { Universidad Nacional } \\
\text { Intercultural de la Amazonía } \\
\text { (UNIA) }\end{array}$ & $\begin{array}{l}\text { Ciudad de Pucallpa } \\
\text { (Región Ucayali) }\end{array}$ & http://unia.edu.pe \\
\hline $\begin{array}{l}\text { Universidad Nacional } \\
\text { Intercultural Fabiola Salazar } \\
\text { Leguía de Bagua (UNIBAGUA) }\end{array}$ & $\begin{array}{l}\text { Provincia de Bagua } \\
\text { (Región Amazonas) }\end{array}$ & http://unibagua.edu.pe \\
\hline
\end{tabular}

Fuente: Elaboración propia.

Según Saquero (2019) estudiaremos qué herramientas se están ofreciendo para garantizar una buena UX y qué es lo que evalúa, ya que, al ser un campo tan grande que abarca desde los colores hasta los sentimientos.

Es común que se confundan los conceptos de interfaz de usuario y experiencia de usuario (UI y UX, respectivamente, por sus siglas en inglés) (Ramírez-Acosta, 2017).

\section{Resultados}

Las universidades nacionales interculturales peruanas cuentan con un sitio web activo, para entregar información a la comunidad universitaria. Pero, debemos indicar que, existen diferencias entre sus sitios web. A continuación, vamos a analizar los resultados obtenidos, después de emplear las herramientas de evaluación del sitio web. El análisis se realizó durante el mes de enero de 2020. 
REVISTA DE LA UNIVERSIDAD DEL ZULIA. $3^{a}$ época. Año $11 N^{\circ}$ 29, 2020

Carlos Ríos Campos et al. /// Análisis de los sitios web de las Universidades ...307-327

Tabla 2. Herramientas para evaluar el sitio web.

\begin{tabular}{|c|c|c|}
\hline Herramientas & Objetivo & Url \\
\hline $\begin{array}{l}\text { PageSpeed } \\
\text { Insights de } \\
\text { Google }\end{array}$ & $\begin{array}{l}\text { Evaluar la velocidad de la } \\
\text { página web e informa sobre el } \\
\text { rendimiento real de una } \\
\text { página para dispositivos } \\
\text { móviles y de escritorio además } \\
\text { de ofrecer sugerencias para } \\
\text { mejorarla. Las métricas se } \\
\text { basan en el Informe de } \\
\text { Experiencia de Usuario de } \\
\text { Chrome. Informa sobre la } \\
\text { velocidad de la página y sobre } \\
\text { la optimización de esta } \\
\text { (PageSpeed Insight, 2020). }\end{array}$ & $\begin{array}{l}\text { https://developers.google.com/speed/ } \\
\text { pagespeed/insights/?hl=es }\end{array}$ \\
\hline $\begin{array}{l}\text { Bankia } \\
\text { Índicex }\end{array}$ & $\begin{array}{l}\text { Analiza el nivel de } \\
\text { competitividad digital de } \\
\text { cualquier negocio a partir de } \\
\text { la evaluación de su } \\
\text { comportamiento en nueve } \\
\text { áreas específicas a través de } \\
\text { más de } 100 \text { parámetros. Estas } \\
\text { áreas son: posicionamiento } \\
\text { SEO, movilidad, experiencia } \\
\text { de usuario, seguridad, } \\
\text { marketing digital, contenidos, } \\
\text { redes sociales, e-commerce y } \\
\text { analítica web (Bankia } \\
\text { Indicex, 2020). }\end{array}$ & https://bankiaindicex.com/ \\
\hline $\begin{array}{l}\text { W3C Markup } \\
\text { Validator }\end{array}$ & $\begin{array}{l}\text { Verifica la validez de marcado } \\
\text { de los documentos Web en } \\
\text { HTML, XHTML, SMIL, } \\
\text { MathML, etc. (W3C Markup } \\
\text { Validator, 2020). }\end{array}$ & https://validator.w3.org/ \\
\hline $\begin{array}{l}\text { Website } \\
\text { Grader }\end{array}$ & $\begin{array}{l}\text { Analiza el rendimiento, la } \\
\text { adaptabilidad móvil, el SEO y } \\
\text { la seguridad de una web } \\
\text { (Website Grader, 2020). }\end{array}$ & https://website.grader.com/ \\
\hline
\end{tabular}


REVISTA DE LA UNIVERSIDAD DEL ZULIA. $3^{2}$ época. Año 11 N² 29, 2020

Carlos Ríos Campos et al. /// Análisis de los sitios web de las Universidades ...307-327

\begin{tabular}{|l|l|l|}
\hline GTMetrix & $\begin{array}{l}\text { Analiza de manera específica } \\
\text { distintos tiempos de carga }\end{array}$ & https://gtmetrix.com/ \\
como el tiempo exacto de cada & \\
request (petición), la & \\
optimización de los recursos, \\
etc. (GTMetrix, 2020).
\end{tabular}

Fuente: Elaboración propia.

\section{2.l. PageSpeed Insights de Google}

Overview
? Scoring Guides
Lighthouse v2 Scoring Guide
Lighthouse v3 Scoring Guide
Lighthouse v3 Migration Guide
Audit References
' Performance
' Progressive Web App
' Accessibility
' Best Practices
, SEO

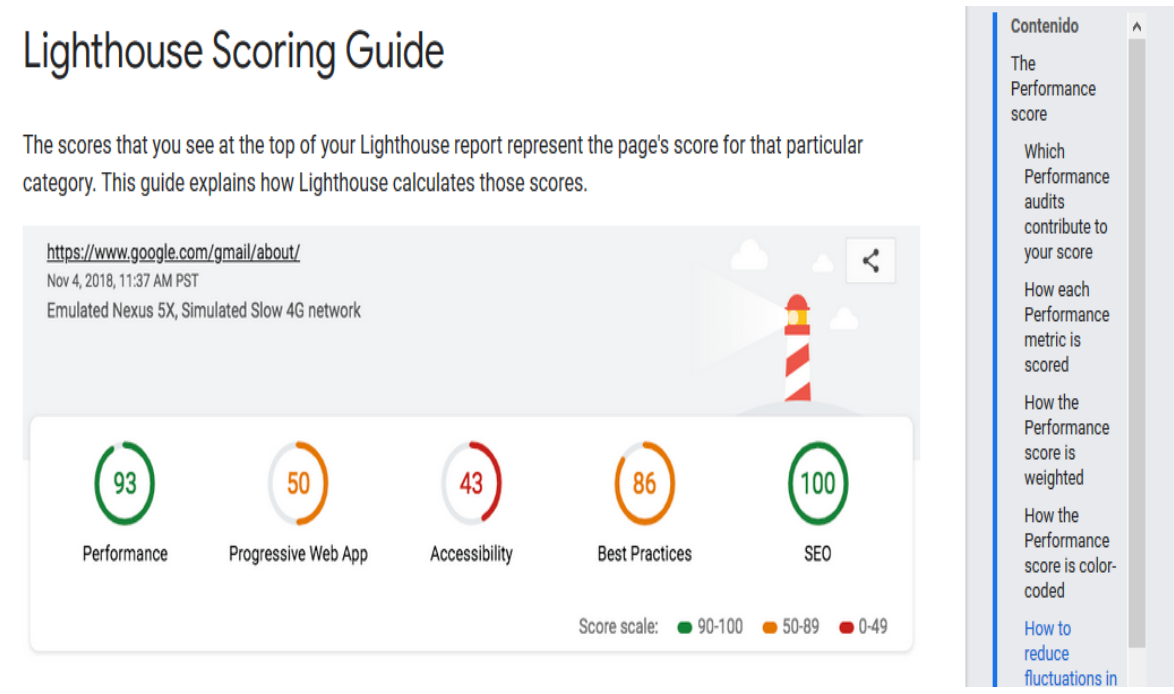

Figura l. Guía de puntuación

Fuente: PageSpeed Insights de Google 
REVISTA DE LA UNIVERSIDAD DEL ZULIA. $3^{a}$ época. Año 11 N 29, 2020 Carlos Ríos Campos et al. /// Análisis de los sitios web de las Universidades ...307-327

2.1.l. Universidad Nacional Intercultural de la Selva Central Juan Santos Atahualpa (UNISCJSA): http://www.uniscjsa.edu.pe

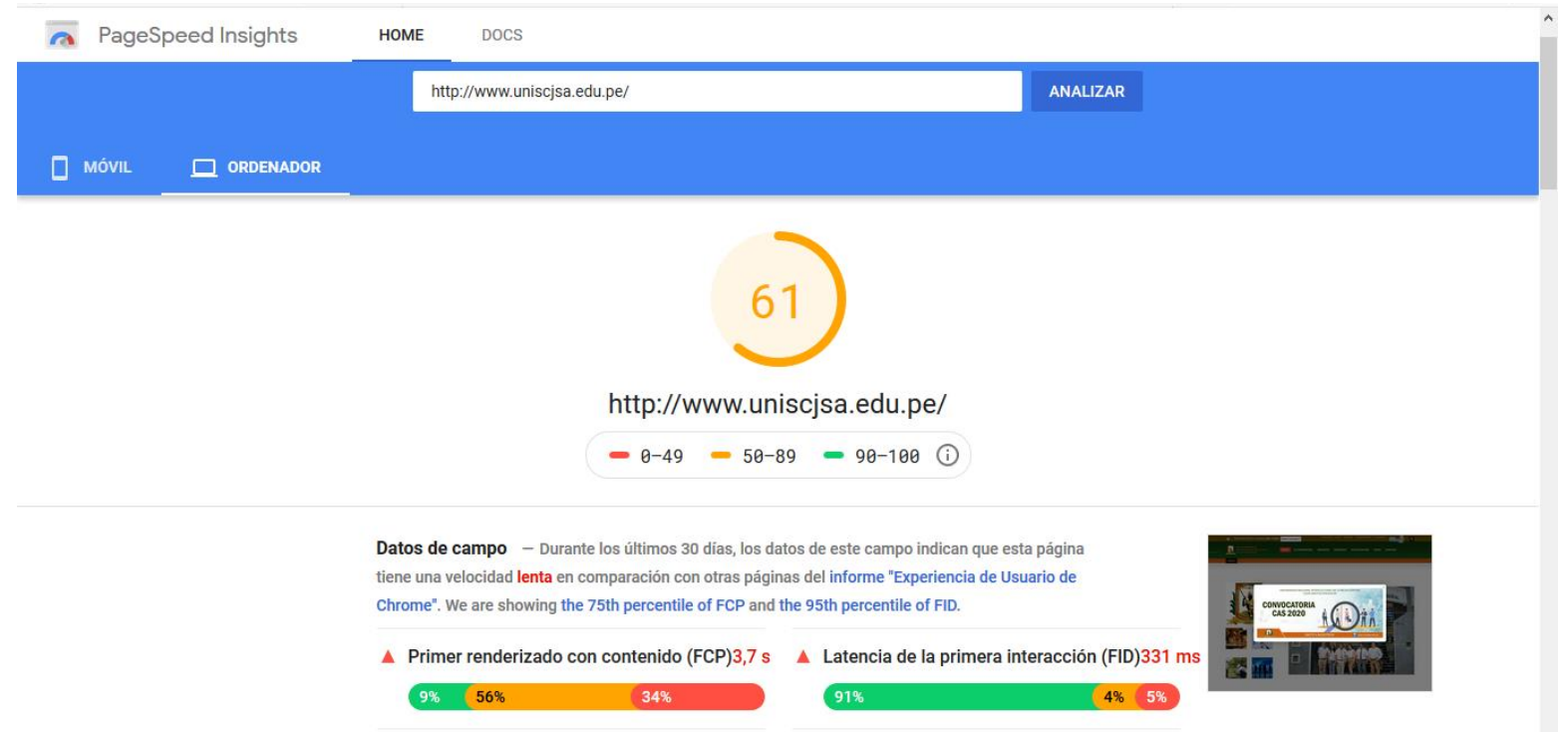

Figura 2. Resultados UNISCJSA

Fuente: PageSpeed Insights de Google

2.1.2. Universidad Nacional Intercultural de Quillabamba (UNIQ): http://www.uniq.edu.pe

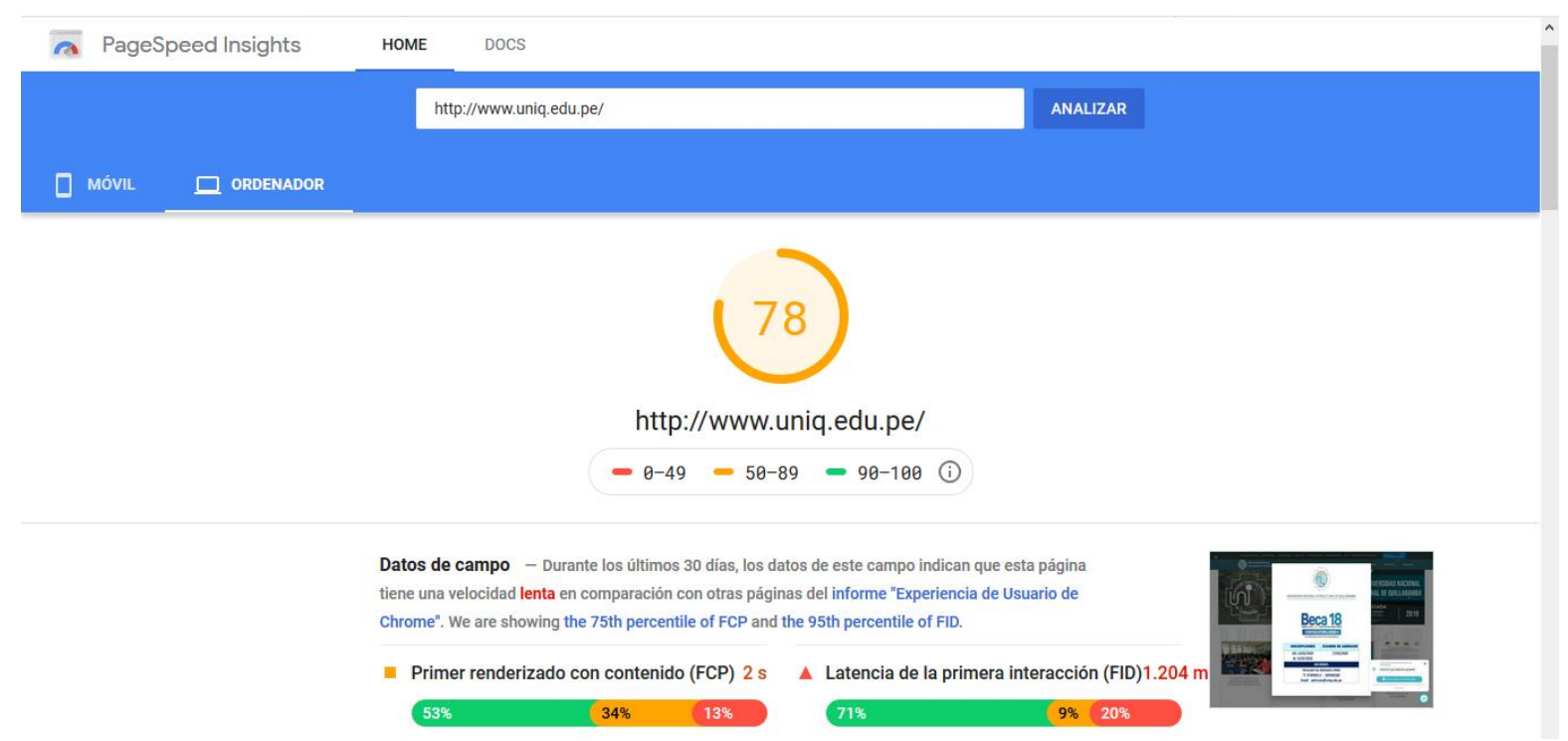

Figura 3. Resultados UNIQ

Fuente: PageSpeed Insights de Google 
REVISTA DE LA UNIVERSIDAD DEL ZULIA. $3^{a}$ época. Año $11 N^{\circ}$ 29, 2020

Carlos Ríos Campos et al. /// Análisis de los sitios web de las Universidades ...307-327

2.1.3. Universidad Nacional Intercultural de la Amazonía (UNIA): http://unia.edu.pe

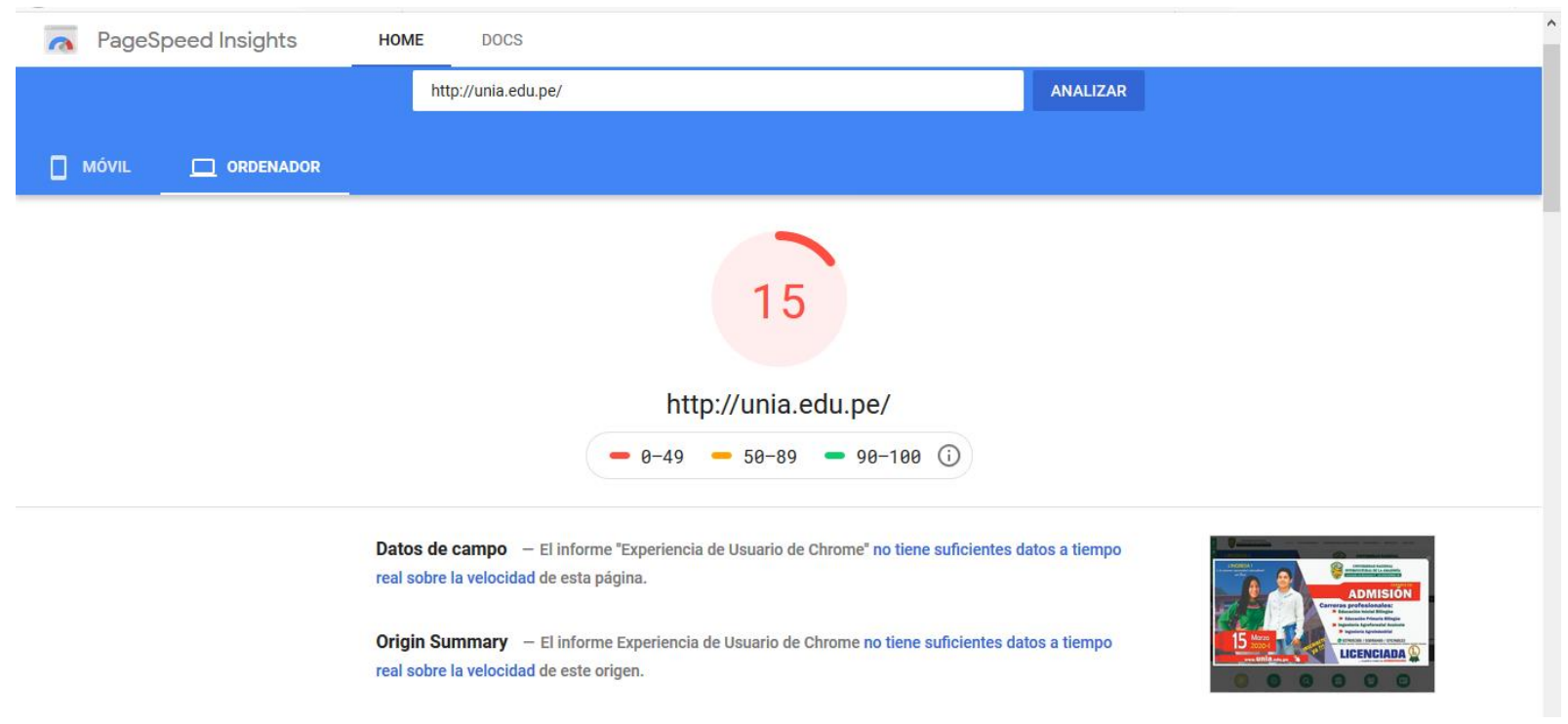

Figura 4. Resultados UNIA

Fuente: PageSpeed Insights de Google

2.1.4. Universidad Nacional Intercultural Fabiola Salazar Leguía de Bagua (UNIBAGUA): http://unibagua.edu.pe

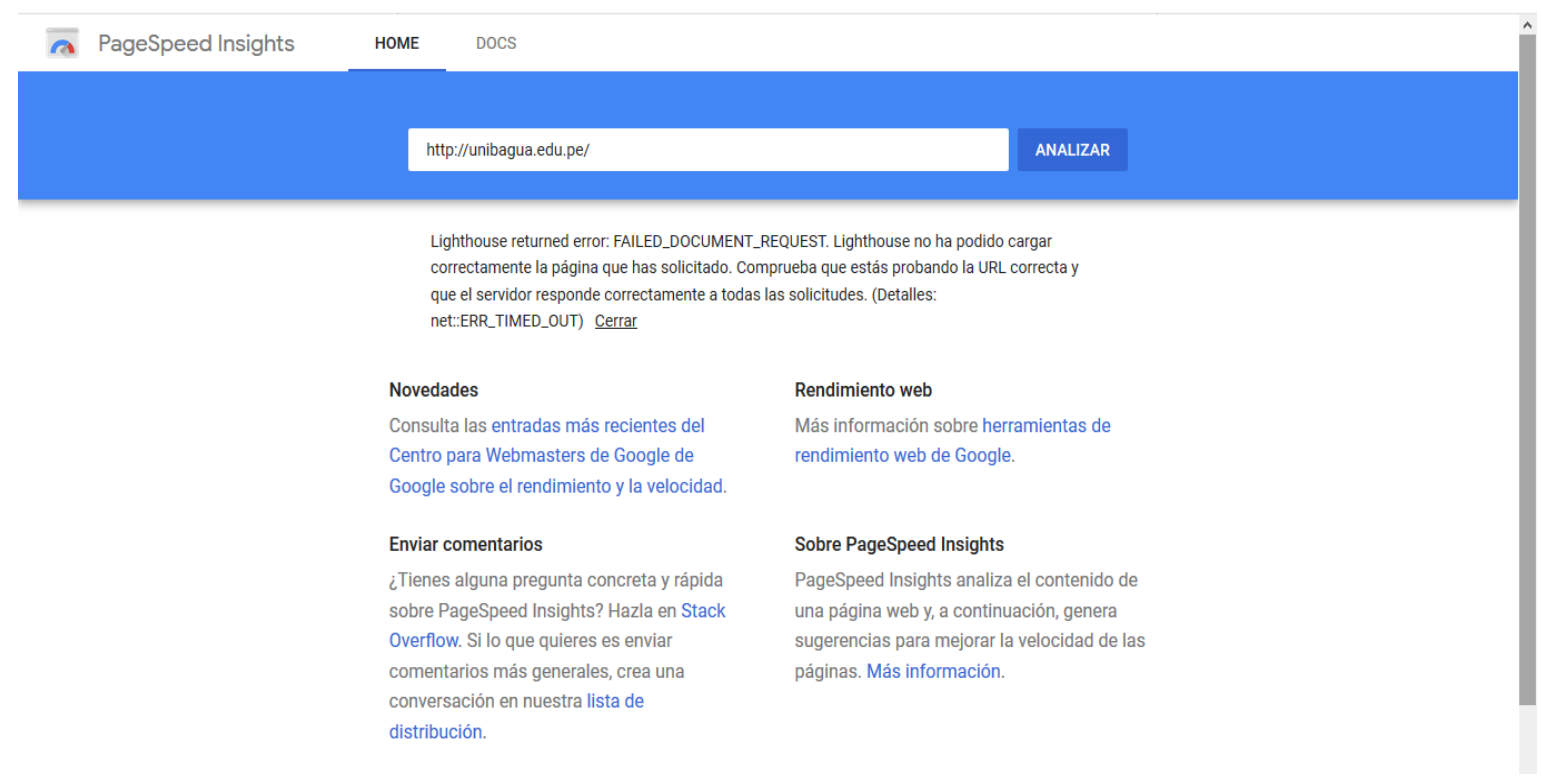

Figura 5. Resultados UNIBAGUA

Fuente: PageSpeed Insights de Google 
REVISTA DE LA UNIVERSIDAD DEL ZULIA. 3a época. Año 11 N$^{\circ}$ 29, 2020 Carlos Ríos Campos et al. /// Análisis de los sitios web de las Universidades ...307-327

Se observó que el mayor puntaje y rendimiento lo tiene la Universidad Nacional Intercultural de Quillabamba (UNIQ) con 78 puntos, mientras que el menor puntaje y rendimiento, corresponde con 15 puntos a la Universidad Nacional Intercultural de la Amazonía (UNIA). Además, se debe indicar que la herramienta no pudo evaluar el sitio web de la Universidad Nacional Intercultural Fabiola Salazar Leguía de Bagua (UNIBAGUA).

2.2. Bankia Índicex

2.2.1. Universidad Nacional Intercultural de la Selva Central Juan Santos Atahualpa (UNISCJSA)

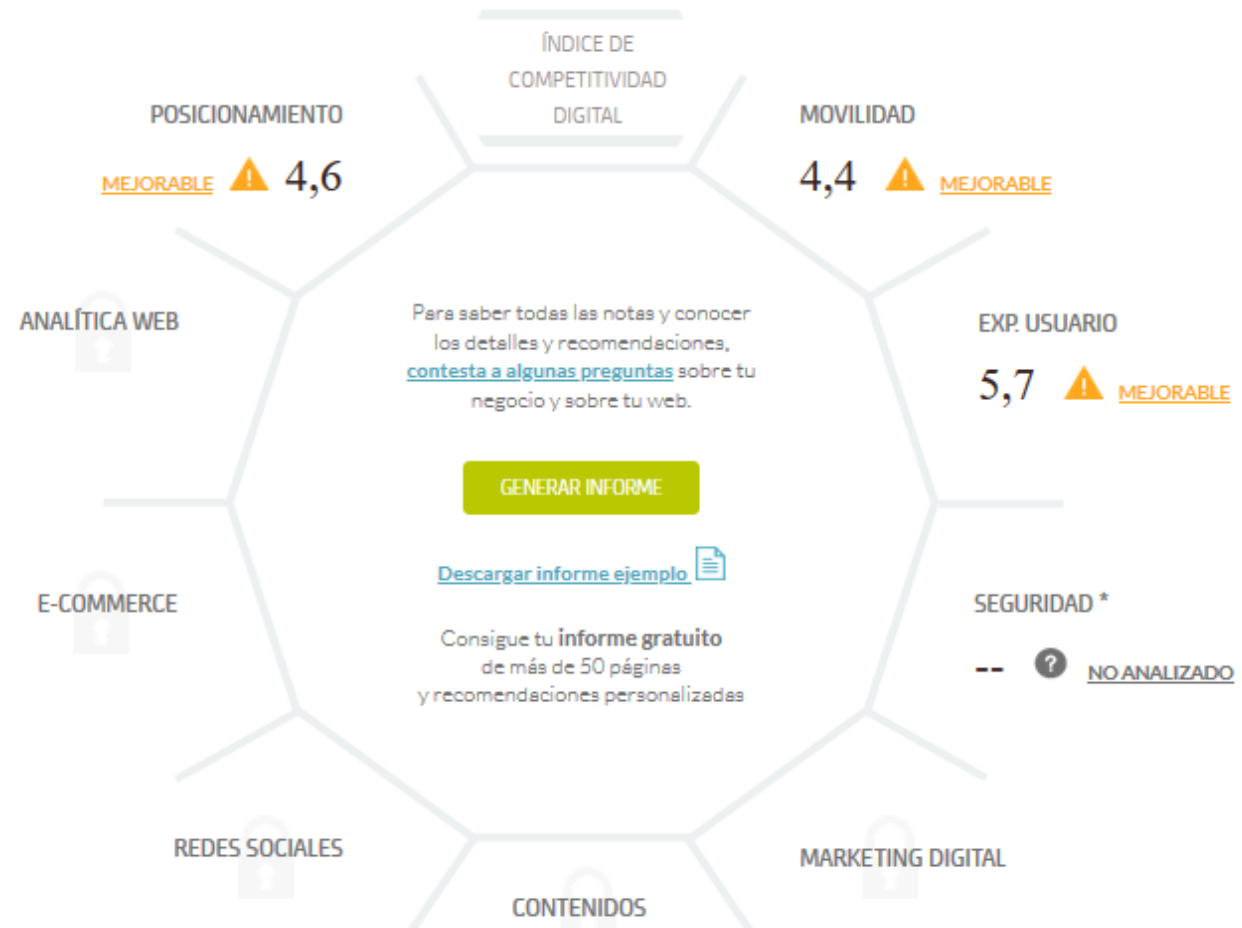

Figura 6. Índice de competitividad digital - UNISCJSA

Fuente: Bankia Índicex 
REVISTA DE LA UNIVERSIDAD DEL ZULIA. 3a época. Año 11 N$^{\circ}$ 29, 2020 Carlos Ríos Campos et al. /// Análisis de los sitios web de las Universidades ...307-327

2.2.2. Universidad Nacional Intercultural de Quillabamba (UNIQ): http://www.uniq.edu.pe

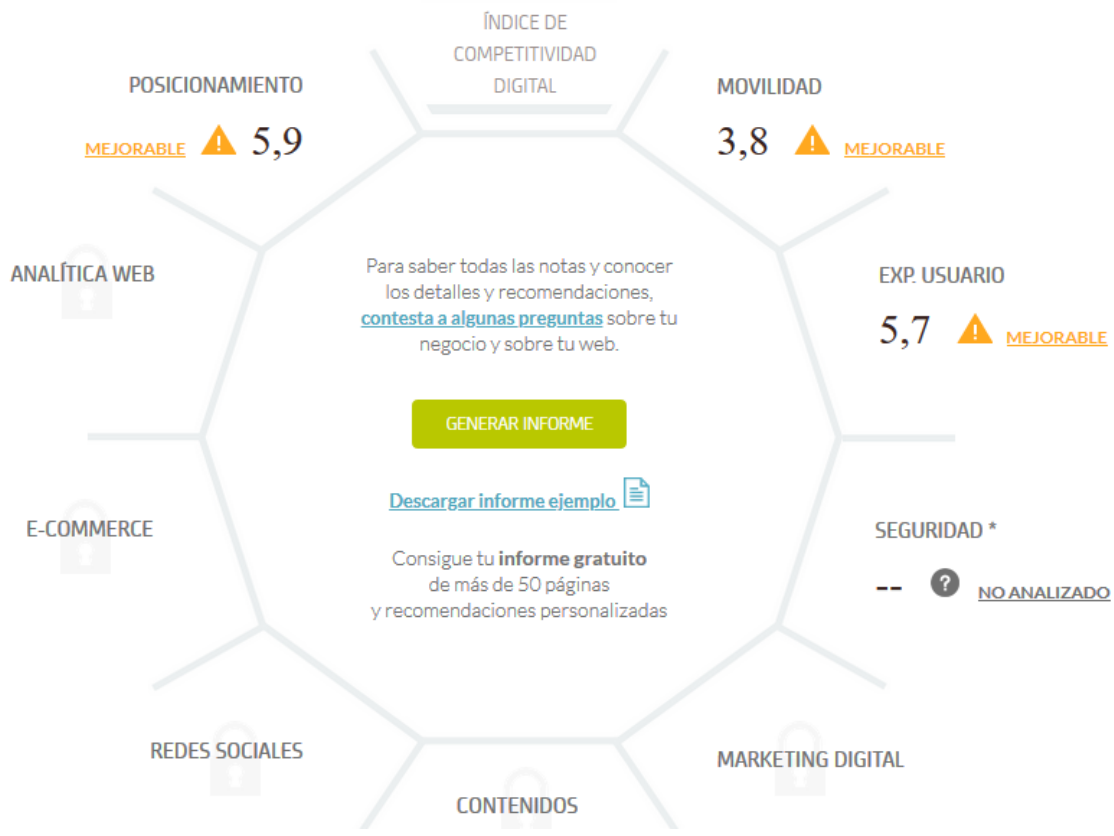

Figura 7. Índice de competitividad digital - UNIQ Fuente: Bankia Índicex

2.2.3. Universidad Nacional Intercultural de la Amazonía (UNIA): http://unia.edu.pe

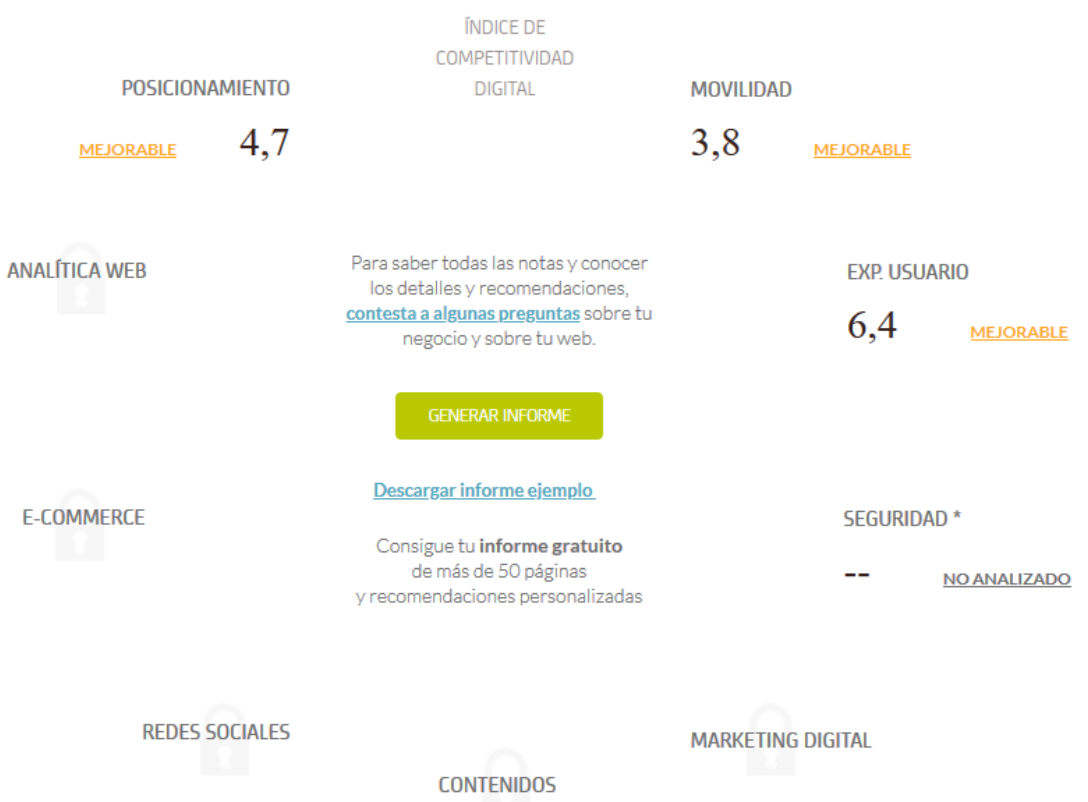

Figura 8. Índice de competitividad digital - UNIA 
REVISTA DE LA UNIVERSIDAD DEL ZULIA. 3a época. Año 11 N$^{\circ}$ 29, 2020

Carlos Ríos Campos et al. /// Análisis de los sitios web de las Universidades ...307-327

Fuente: Bankia Índicex

2.2.4. Universidad Nacional Intercultural Fabiola Salazar Leguía de Bagua (UNIBAGUA): http://unibagua.edu.pe

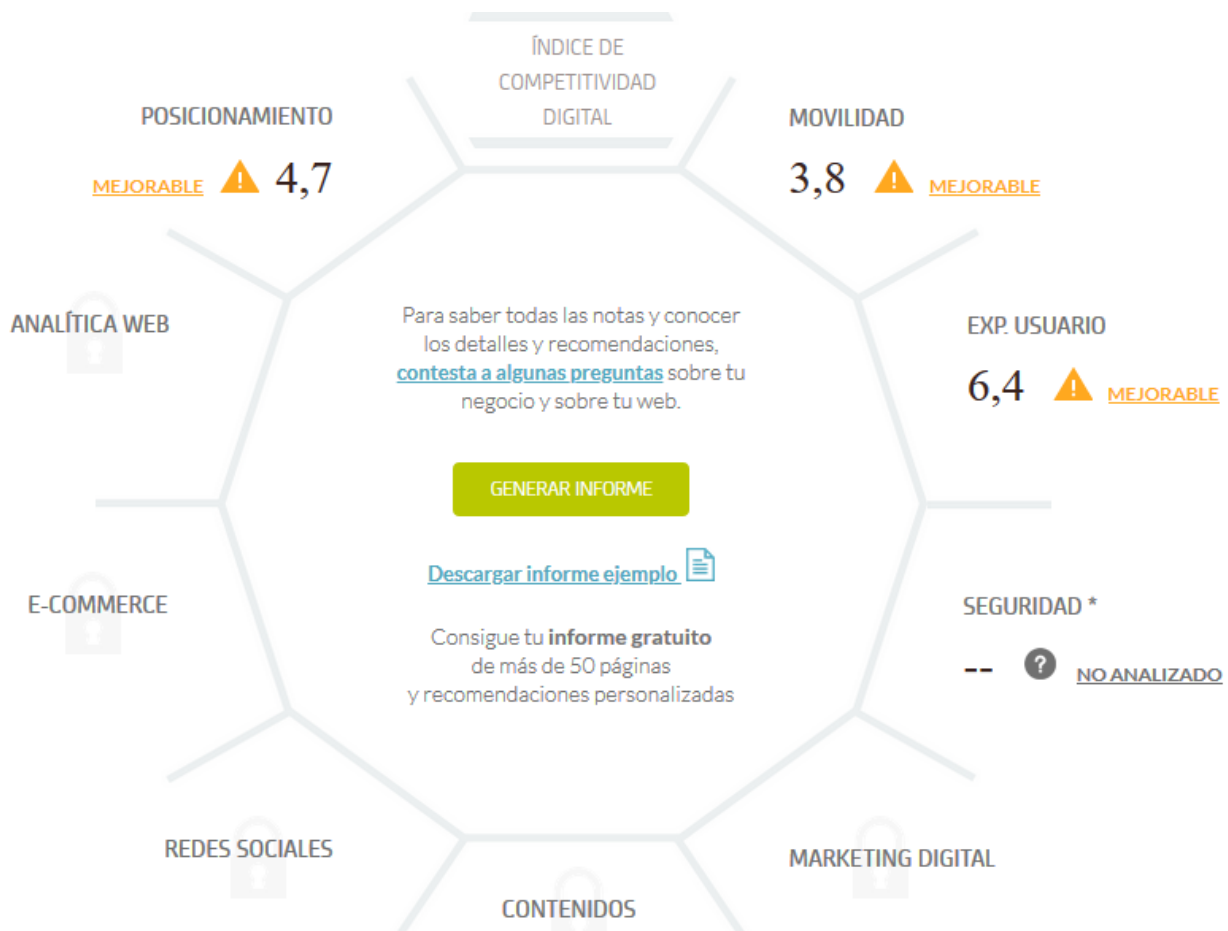

Figura 9. Índice de competitividad digital - UNIBAGUA

Fuente: Bankia Índicex

Se observó que el mayor nivel de posicionamiento lo obtuvo la Universidad Nacional Intercultural de Quillabamba (UNIQ), con 5,9 puntos. El mayor nivel de movilidad lo obtuvo la Universidad Nacional Intercultural de la Selva Central Juan Santos Atahualpa (UNISCJSA) con 4,4 puntos. El mayor nivel de experiencia de usuario lo obtuvieron la Universidad Nacional Intercultural de la Amazonía (UNIA) y la Universidad Nacional Intercultural Fabiola Salazar Leguía de Bagua (UNIBAGUA) con 6,4 puntos.

\subsection{W3C Markup Validator}

2.3.1. Universidad Nacional Intercultural de la Selva Central Juan Santos Atahualpa (UNISCJSA): http://www.uniscjsa.edu.pe 
REVISTA DE LA UNIVERSIDAD DEL ZULIA. $3^{2}$ época. Año 11 N²9, 2020

Carlos Ríos Campos et al. /// Análisis de los sitios web de las Universidades ...307-327

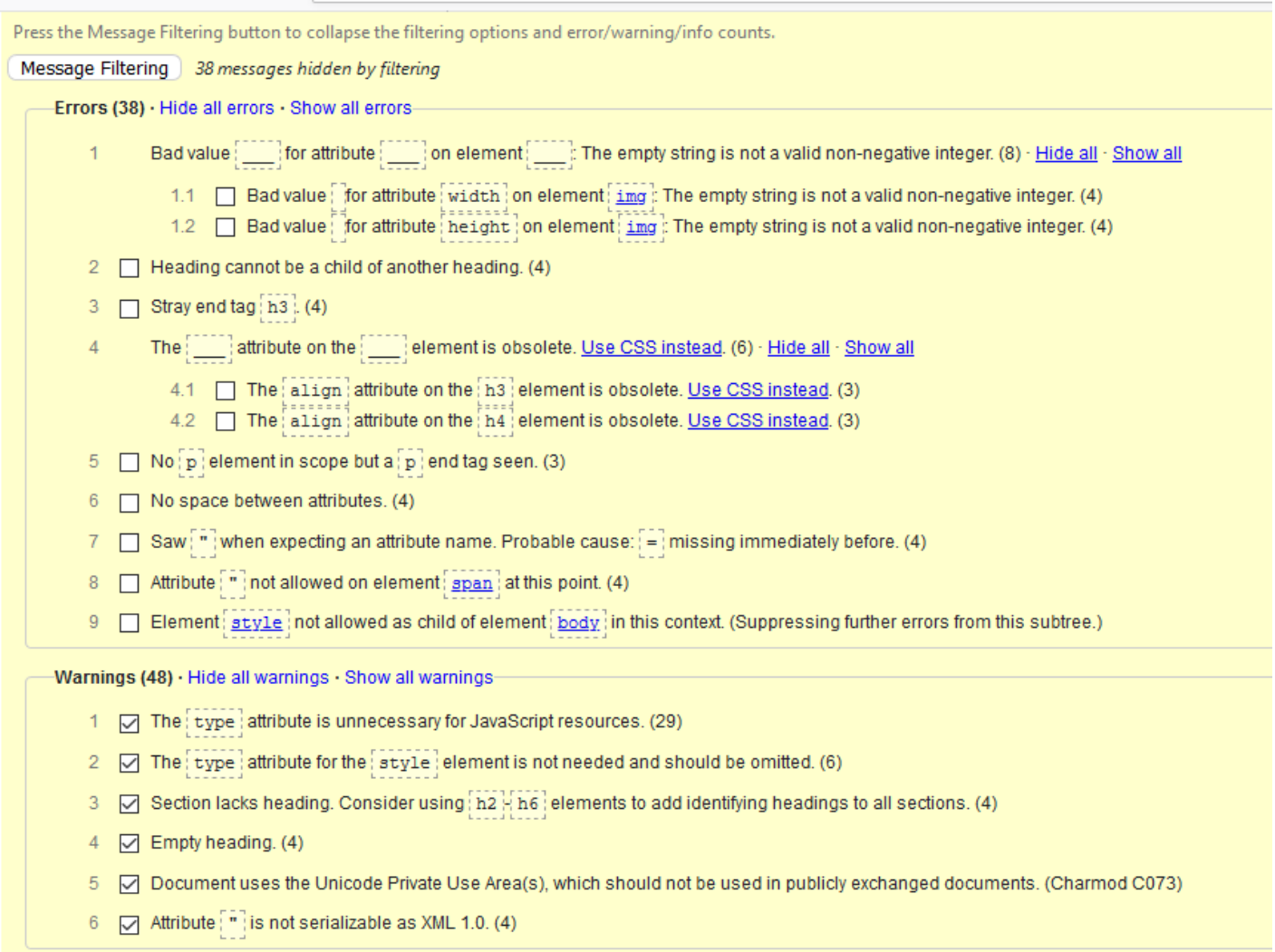

Figura 10. Validar un documento en línea - UNISCJSA Fuente: W3C Markup Validator

\subsubsection{Universidad Nacional Intercultural de Quillabamba (UNIQ): http://www.uniq.edu.pe}

Press the Message Filtering button to collapse the filtering options and error/warning/info counts. Message Filtering

Errors (5) $\cdot$ Hide all errors $\cdot$ Show all errors

$1 \quad$ Attribute $\quad$ not allowed on element

$1.1 \square$ Attribute attribution not allowed on element div at this point.

$1.2 \square$ Attribute page_id not allowed on element

$1.3 \square$ Attribute theme_color not allowed on element div at this point.

$1.4 \square$ Attribute logged_in_greeting not allowed on element

$1.5 \square$ Attribute logged_out_greeting not allowed on element div at this point.

Warnings (1) $\cdot$ Hide all warnings $\cdot$ Show all warnings

$1 \square$ The type attribute is unnecessary for JavaScript resources.

Figura 1l. Validar un documento en línea - UNIQ Fuente: W3C Markup Validator 
REVISTA DE LA UNIVERSIDAD DEL ZULIA. $3^{a}$ época. Año 11 N 29, 2020

Carlos Ríos Campos et al. /// Análisis de los sitios web de las Universidades ...307-327

2.3.3. Universidad Nacional Intercultural de la Amazonía (UNIA): http://unia.edu.pe

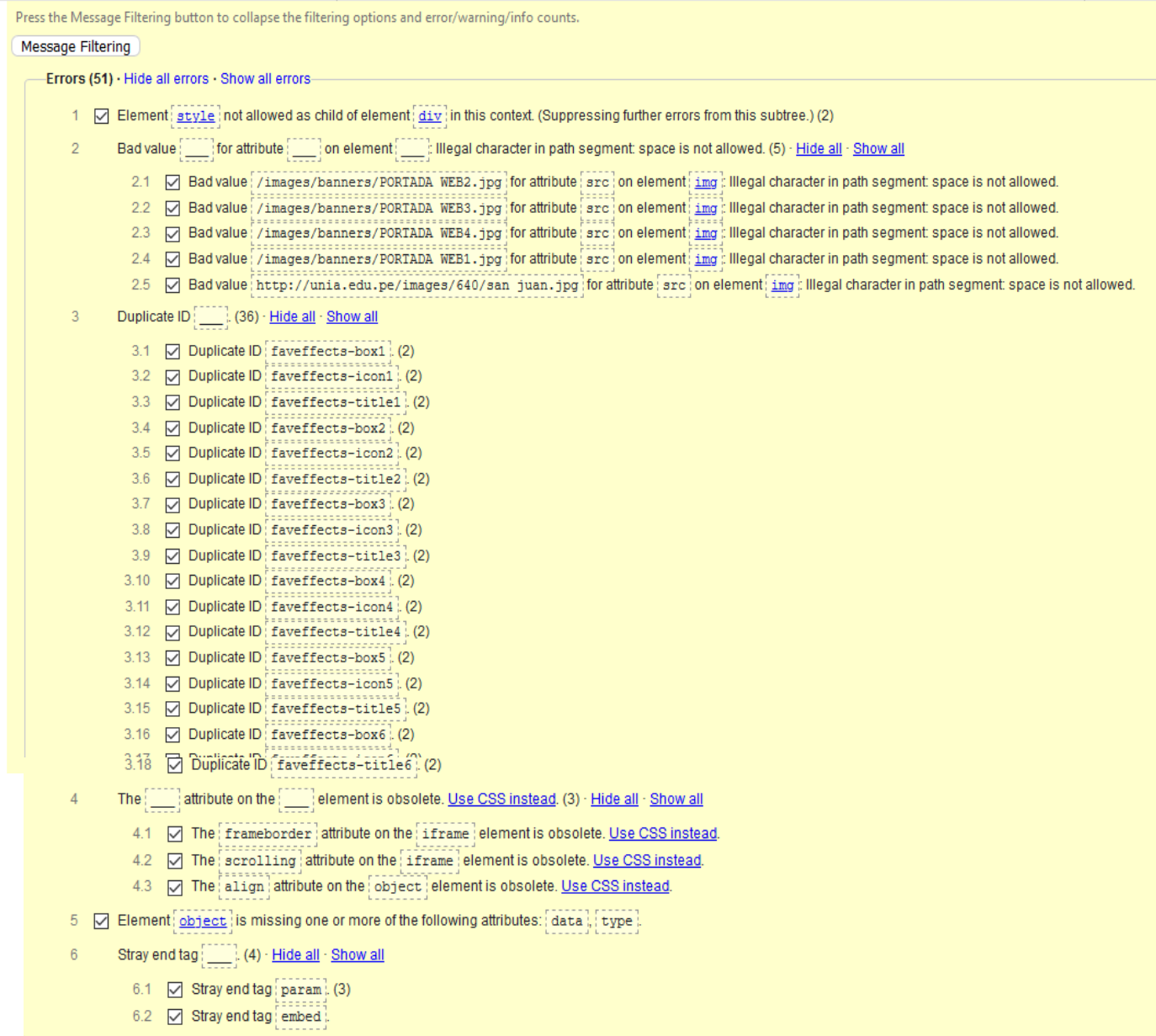

Warnings (98) · Hide all warnings · Show all warnings

$1 \square$ The type attribute for the style element is not needed and should be omitted. (3)

$2 \square$ The type attribute is unnecessary for JavaScript resources. (27)

The first occurrence of ID $[\ldots$ was here. (36) - Hide all - Show all

$3.1 \square$ The first occurrence of ID faveffects-box 1 was here. (2)

2 The first occurrence of ID faveffects-icon 1 was here. (2)

3.3 The first occurrence of ID faveffects-title1 was here. (2)

$34 \square$ The first occurrence of ID faveffects-box 2 was here. (2)

3.5 The first occurrence of ID faveffects-icon2 was here. (2)

3.6 $\square$ The first occurrence of ID faveffects-title2 was here. (2

$3.7 \square$ The first occurrence of ID faveffects-box3 : was here. (2)

3.8 $\square$ The first occurrence of ID faveffects-icons w was here. (2)

$3.9 \square$ The first occurrence of ID faveffects-title 3 ; was here. (2)

3.10 $\square$ The first occurrence of ID faveffect-box 4 ; was here. (2)

3.11 $\square$ The first occurrence of ID faveffects-icon 4 was here. (2)

$3.12 \nabla$ The first occurrence of ID faveffects-title 4 : was here. (2)

3.13 $\square$ The first occurrence of ID faveffects-box5 was here. (2)

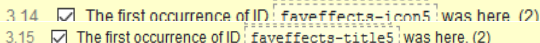

316 The first occurrence of ID faveffects-box 6 : was here (2)

317 The first occurrence of ID : faveffect $3-1$ icon6 ? was here. (2)

3.18 The first occurrence of ID faveffects-title6 : was here. (2)

$4 \square$ Section lacks heading. Consider using $\mathrm{h} 2 \mathrm{~h} 6$ elements to add identifying headings to all sections.

$5 \square$ The border attribute is obsolete. Consider specifying ing i border: $0 ; 1$ in Css instead. (31)

Figura 12. Validar un documento en línea - UNIA 
REVISTA DE LA UNIVERSIDAD DEL ZULIA. $3^{a}$ época. Año 11 N 29, 2020

Carlos Ríos Campos et al. /// Análisis de los sitios web de las Universidades ...307-327

Fuente: W3C Markup Validator

2.3.4. Universidad Nacional Intercultural Fabiola Salazar Leguía de Bagua (UNIBAGUA): http://unibagua.edu.pe

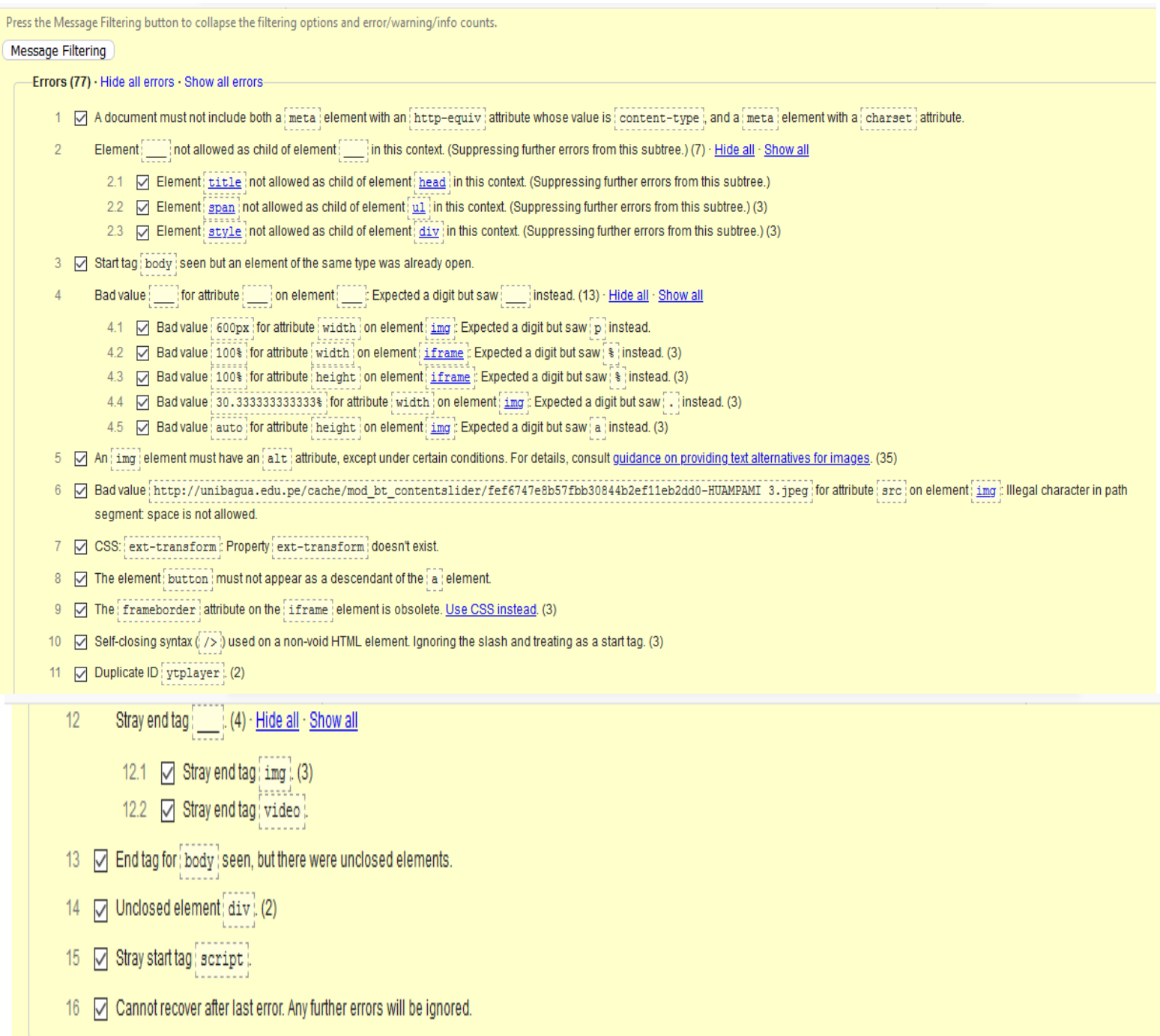

Warnings (38) · Hide all warnings · Show all warnings

$1 \square$ The type attribute for the style element is not needed and should be omitted. (2)

$2 \square$ The type attribute is unnecessany for JavaScipt resources. (30)

$3 \square$ The language attribute on the script element is obsolete. You can safely omitit.

$4 \square$ The navigation role is unnecessary for element nav?

$5 \square$ Section lacks heading. Consider using; $\mathrm{h} 2$, h6 elements to add identifing headings to all sections. (2)

6 凤 The first occurrence of ID vtolaver was here. (2)

Figura 13. Validar un documento en línea - UNIBAGUA Fuente: W3C Markup Validator 
REVISTA DE LA UNIVERSIDAD DEL ZULIA. $3^{2}$ época. Año 11 N² 29, 2020 Carlos Ríos Campos et al. /// Análisis de los sitios web de las Universidades ...307-327

Se observó que el mayor nivel de errores (5l) y advertencias (98) fue el sitio web de la Universidad Nacional Intercultural de la Amazonía (UNIA), mientras que el menor nivel de errores (5) y advertencias (1) fue el sitio web de la Universidad Nacional Intercultural de Quillabamba (UNIQ).

\subsection{Website Grader}

2.4.1. Universidad Nacional Intercultural de la Selva Central Juan Santos Atahualpa (UNISCJSA): http://www.uniscjsa.edu.pe

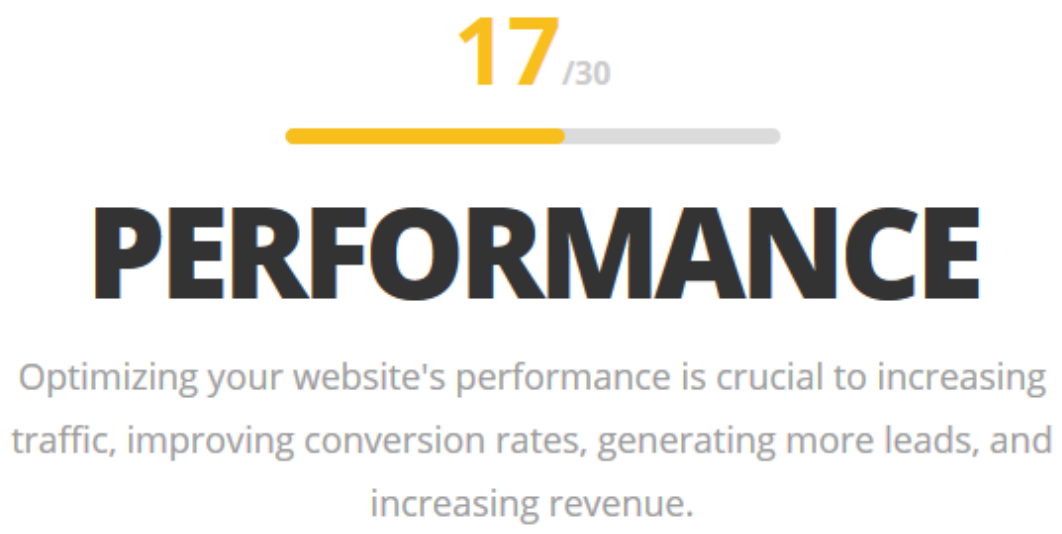

Figura 14. Análisis del rendimiento - UNISCJSA

Fuente: Website Grader

2.4.2. Universidad Nacional Intercultural de Quillabamba (UNIQ): http://www.uniq.edu.pe

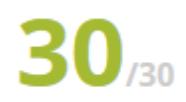

\section{PERFORMANCE}

Optimizing your website's performance is crucial to increasing traffic, improving conversion rates, generating more leads, and increasing revenue.

Figura 15. Análisis del rendimiento - UNIQ Fuente: Website Grader 
REVISTA DE LA UNIVERSIDAD DEL ZULIA. $3^{2}$ época. Año 11 N² 29, 2020 Carlos Ríos Campos et al. /// Análisis de los sitios web de las Universidades ...307-327

2.4.3. Universidad Nacional Intercultural de la Amazonía (UNIA): http://unia.edu.pe

\section{9

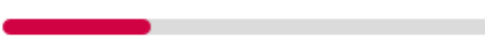 \\ PERFORMANCE}

Optimizing your website's performance is crucial to increasing

traffic, improving conversion rates, generating more leads, and

increasing revenue.

Figura 16. Análisis del rendimiento - UNIA

Fuente: Website Grader

2.4.4. Universidad Nacional Intercultural Fabiola Salazar Leguía de Bagua (UNIBAGUA): http://unibagua.edu.pe

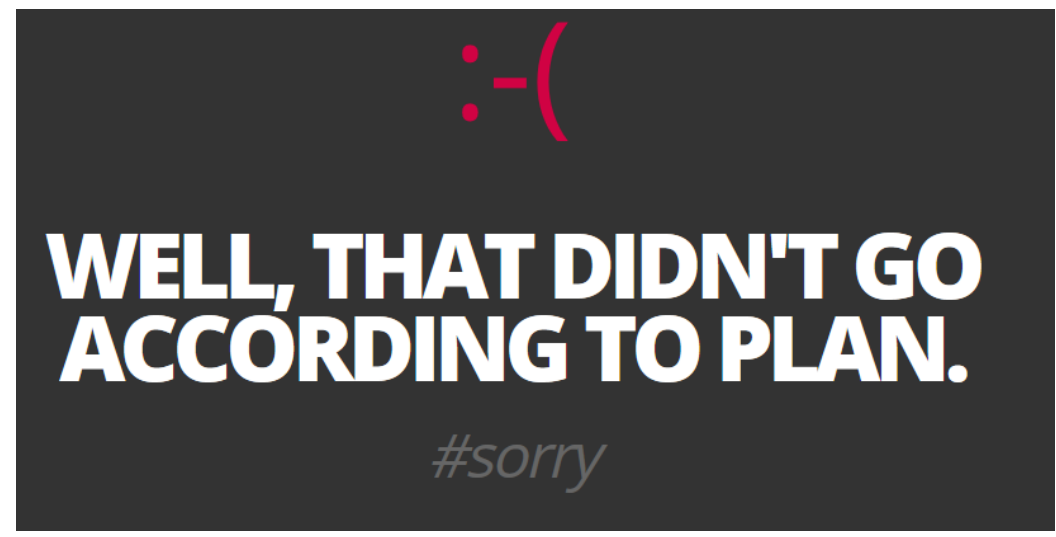

Figura 17. Análisis del rendimiento - UNIBAGUA

Fuente: Website Grader

Se observó que el mejor rendimiento lo tiene el sitio web de la Universidad Nacional Intercultural de Quillabamba (UNIQ) con 30 puntos y el peor rendimiento tiene el sitio web de la Universidad Nacional Intercultural Fabiola Salazar Leguía de Bagua (UNIBAGUA), pues ni siquiera permite a la herramienta realizar el análisis respectivo. 
REVISTA DE LA UNIVERSIDAD DEL ZULIA. $3^{2}$ época. Año 11 N²9, 2020

Carlos Ríos Campos et al. /// Análisis de los sitios web de las Universidades ...307-327

\subsection{GTMetrix}

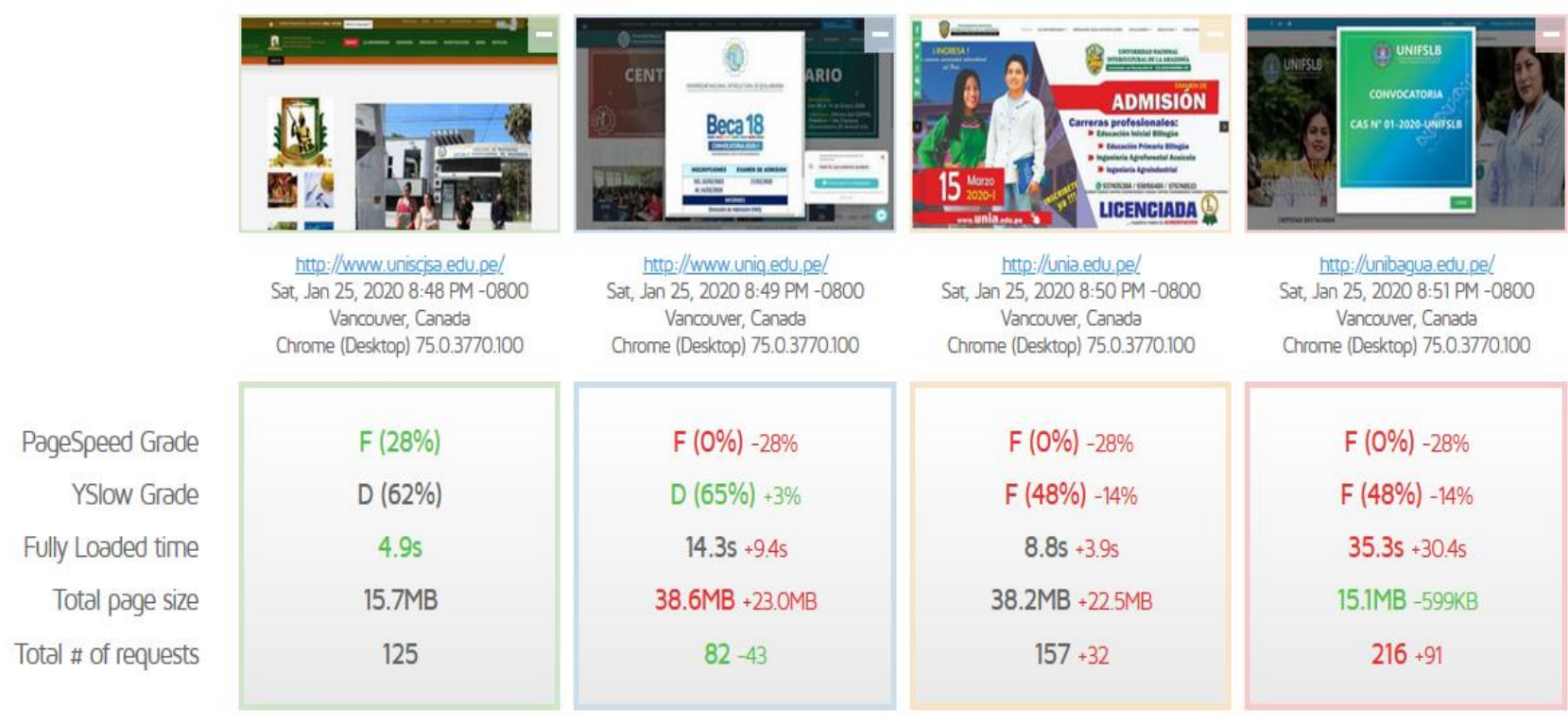

Figura 18. Análisis de tiempos de carga - UNISCJSA Fuente: GTMetrix

Se observó que el mejor tiempo de carga lo tiene el sitio web de la Universidad Nacional Intercultural de la Selva Central Juan Santos Atahualpa (UNISCJSA) con $4.9 \mathrm{~s}$, mientras que el peor tiempo de carga lo tiene el sitio web de la Universidad Nacional Intercultural Fabiola Salazar Leguía de Bagua (UNIBAGUA) con 35.3 s.

\section{Discusión}

A tenor de los resultados obtenidos en la investigación, se coincide con (Calvo $\&$ Riu, 2019) quien afirma que, el testeo de la propuesta de análisis para sitios Web ha permitido comprobar que la Web se mantiene como el principal eje comunicativo de las entidades.

Se evidencia que las características de los sitios web de las cuatro universidades interculturales, ofrecen un canal de comunicación gratuito y rápido con la comunidad universitaria, por lo tanto, se coincide con (Fuertes \& Martínez, 2017), quien afirma que, en este sentido es esencial que la web facilite la integración de todas las personas 
REVISTA DE LA UNIVERSIDAD DEL ZULIA. $3^{a}$ época. Año $11 N^{\circ}$ 29, 2020

Carlos Ríos Campos et al. /// Análisis de los sitios web de las Universidades ...307-327

en esta nueva sociedad, evitando discriminar por razones de edad, conocimientos, idioma, formación, tecnología, cultura, religión, género y, por supuesto, discapacidad.

\section{Conclusiones}

Con respecto al primer objetivo, determinar los problemas frecuentes que presentan los sitios web de las universidades nacionales interculturales peruanas: Se concluyó que, el mayor nivel de errores (51) y advertencias (98) fue el sitio web de la Universidad Nacional Intercultural de la Amazonía (UNIA), mientras que el menor nivel de errores (5) y advertencias (1) fue el sitio web de la Universidad Nacional Intercultural de Quillabamba (UNIQ).

En relación con el segundo objetivo, identificar si existen diferencias específicas entre los sitios web de las universidades nacionales interculturales peruanas: Se concluyó que, el mayor puntaje y rendimiento lo tiene la Universidad Nacional Intercultural de Quillabamba (UNIQ) con 78 puntos. Además, se debe indicar que la herramienta no pudo evaluar el sitio web de la Universidad Nacional Intercultural Fabiola Salazar Leguía de Bagua (UNIBAGUA). El mayor nivel de posicionamiento lo obtuvo la Universidad Nacional Intercultural de Quillabamba (UNIQ), con 5,9 puntos. El mayor nivel de movilidad lo obtuvo la Universidad Nacional Intercultural de la Selva Central Juan Santos Atahualpa (UNISCJSA) con 4,4 puntos. El mayor nivel de experiencia de usuario lo obtuvieron la Universidad Nacional Intercultural de la Amazonía (UNIA) y la Universidad Nacional Intercultural Fabiola Salazar Leguía de Bagua (UNIBAGUA) con 6,4 puntos. El mejor rendimiento lo tiene el sitio web de la Universidad Nacional Intercultural de Quillabamba (UNIQ) con 30 puntos. El mejor tiempo de carga lo tiene el sitio web de la Universidad Nacional Intercultural de la Selva Central Juan Santos Atahualpa (UNISCJSA) con 4.9 s.

En cuanto al tercer objetivo, examinar las ventajas importantes que ofrecen los sitios web a las universidades nacionales interculturales peruanas: Se concluyó que, facilitan la comunicación fluida con la comunidad universitaria, empleando el Internet como medio de acceso económico y amplio. 
REVISTA DE LA UNIVERSIDAD DEL ZULIA. $3^{2}$ época. Año 11 N²9, 2020

Carlos Ríos Campos et al. /// Análisis de los sitios web de las Universidades ...307-327

\section{Referencias}

Aguilar, D. (2019). Sociedad del conocimiento y el entorno digital. Revista Latinoamericana de Ensayo. Recuperado de http://critica.cl/educacion/sociedad-del-conocimiento-y-elentorno-digital

Alonso-García, S., Roque-Herrera, Y., \& Juárez-Ramos, V. (2019). La educación intercultural en el contexto ecuatoriano de educación superior: un caso de innovación curricular. Tendencias Pedagógicas, 33, 47-58. doi: 10.15366/tp2019.33.004

Bankia Índicex (2019). Bankia Índicex: Observatorio de la transformación digital. Recuperado de https://bankiaindicex.com/

Calvo, S. \& Riu, L. (2019). Propuesta teórica para la sistematización, archivo y recuperación de los datos de los sitios Web de entidades del tercer sector: El caso Colombia - Cataluña. Transinformação, 31, el80069. Epub March 18, 2019.https:/dx.doi.org/10.1590/23180889201931el80069

Católico-Segura, D., Leal-Bonilla, K. \& Londoño-Jiménez, O. (2019). La transparencia activa y su vínculo con el origen institucional: el caso de las universidades colombianas. Revista iberoamericana de educación superior, 10(27), 51-71. https://dx.doi.org/10.22201/iisue.20072872e.2019.27.340

Espinosa, Oscar. (2017). Educación superior para indígenas de la Amazonía peruana: balance y desafios. Anthropologica, $35(39)$, $99-122$. https://dx.doi.org/10.18800/anthropologica.201702.005

Ferrer, T. (2017). Las Tecnologías de la Información y Comunicación, ¿el nuevo paradigma para acceder al conocimiento?, Revista de la Universidad del Zulia, 8 (20), 9-10. https://produccioncientificaluz.org/index.php/rluz/article/view/30881

Fuertes, J., \& Martínez, L. (2017). Accesibilidad Web. TRANS. Revista de Traductología, O(11), 135-154. doi:http://dx.doi.org/10.24310/TRANS.2007.v0ill.3103

GTMetrix (2020). How fast does your website load?. Find out with GTmetrix. Recuperado de https:/gtmetrix.com/

Hernandez, R. (2017). Impacto de las TIC en la educación: Retos y Perspectivas. Propósitos y Representaciones, 5(1), 325 - 347 http://dx.doi.org/10.2051l/pyr2017.v5nl.149

Maldonado, G., García, J., y Sampedro-Requena, B. (2019). El efecto de las TIC y redes sociales en estudiantes universitarios. RIED. Revista Iberoamericana de Educación a Distancia, 22(2), pp. 153-176. doi: http://dx.doi.org/10.5944/ried.22.2.23178

Martínez, C. (2019). Responsabilidad social universitaria, transferencia tecnológica y desarrollo endógeno. Estrategias de vinculación comunitaria, Revista 
REVISTA DE LA UNIVERSIDAD DEL ZULIA. $3^{2}$ época. Año 11 N²9, 2020 Carlos Ríos Campos et al. /// Análisis de los sitios web de las Universidades ...307-327

Latinoamericana de Difusión Científica, 1 (1), 55-67. http://difusioncientifica.info/index.php/difusioncientifica/article/view/7

PageSpeed Insight (2019). Acerca de PageSpeed Insights. Recuperado de https://developers.google.com/speed/docs/insights/about

Ramírez-Acosta, K. (2017). Interfazy experiencia de usuario: parámetros importantes para un diseño efectivo. Revista Tecnología en Marcha, 30(Suppl. 1), 49-54. https://dx.doi.org/10.18845/tm.v30i5.3223

Rodríguez, R. (2014). Internet, salud pública 2.0 y complejidad. Revista de la Universidad Industrial de Santander. Salud, 46(3), 297-303. Recuperado de http://www.scielo.org.co/scielo.php?script=sci_arttext\&epid=SO121$08072014000300010 \&$ lng=en\&tlng=es.

Rovira, C. y Marcos, M. (2013). Diseño de sitios web: disciplinas, materias y esquemas integradores. Hipertext.net, $12 . \quad$ Recuperado de http://www.upf.edu/hipertextnet/numero_ll/Diseno_sitios_web.html

Rueda, G., Paz, L. \& Avendaño, W. (2019). Análisis de la Educación Intercultural en Grupos de Estudiantes de la Universidad Francisco de Paula Santander, en Colombia, que Fueron Víctimas del Conflicto Armado. Formación universitaria, 12(4), 95-104. https://dx.doi.org/10.4067/S0718-50062019000400095

Saquero, C. (2019). Diseño y desarrollo de una metodología de evaluación de la UX. (Tesis pregrado). Universidad de Alicante. https://rua.ua.es/dspace/bitstream/10045/88488/1/Diseno_y_desarrollo_de_una_meto dologia_de_evaluacion_del_U_SAQUERO_ROS_CLARA.pdf

UNESCO (2019). Líneas Generales. Recuperado de http://www.unesco.org/new/es/mexico/work-areas/culture/

UNESCO (2017). Interculturalidad. Recuperado de http://www.unesco.org/new/es/quito/education/education-and-interculturality/

UNIA (2020). Historia. Recuperado de https://www.unia.edu.pe/index.php/launiversidad/historia

Website Grader (2020). How strong is your website?. Recuperado de https://website.grader.com/

W3C Markup Validator (2019). Markup Validator Service. Recuperado de https://validator.w3.org/ 